\title{
Localized Approaches for Nonlinear Analysis of Chaotic Systems in Multidimensional Phase Space
}

\author{
Victor F. Dailyudenko*
}

Institute of Informatics Problems NAS of Belarus, Surganov St. 6, 220012, Minsk, Belarus

\begin{abstract}
In this paper, we develop algorithms for calculation of fractal measures and characteristic exponents for modeling of chaotic systems evolution. Using temporal localization along phase trajectories of a chaotic attractor reconstructed from nonlinear time series, we achieve the essential reduction of required computer resources that allows the nonlinear analysis algorithms to be realized even for higher-dimensional cases and provides their robustness to enlarging the length of time series. The numerical simulations confirm reliability of the developed algorithms and their high efficiency at calculation of fractal dimension.
\end{abstract}

Keywords: Nonlinear time series, multidimensional attractors, characteristic exponents, fractal dimensions, numerical algorithms.

\section{INTRODUCTION}

The problem of chaotic systems (CSs) investigation while their temporal evolution simulated on data measured or obtained from respective differential equations has become a problem of great significance in various fields of physics [1], as well as in medicine and engineering [1-3]. Time series (TS) obtained from a CS are essentially nonlinear [1-3]. and often lead to a multidimensional attractor in a relevant phase space [3-7]. Namely, it occurs at investigation of highly-developed turbulence where higherorder modes become important for increasing reliability [7].

The challenge of CS' synchronization deals with their stability and predictability, both characteristic exponents and fractal dimensions describing a CS' attractor and being used at development of synchronization methods [4]. The algorithms of the fractal analysis of chaotic attractors [1-7] involve approximation methods of finite sets consideration and provide an opportunity to detect self-similar properties of CS' temporal evolution through digital processing of phase trajectories data. But it is worth noting that the main problem of numerical analysis of TS in such highdimensional cases is that the computation complexity of nonlinear analysis algorithms essentially increases with enlarging a dimension $m$ (as a rule exponentially [1-5]) that makes them hardly to implement. So, in this paper we develop algorithms providing the essential reduction of computation time and required memory due to the properties of temporal localization in relation to points of an attractor.

\section{THE FRACTAL MEASURES CALCULATION}

For reconstruction of phase trajectories forming an attractor $R_{T}^{m}$, let us use the method of delayed coordinates $[1,5,8]$.

*Address correspondence to this author at the Institute of Informatics Problems NAS of Belarus, Surganov St. 6, 220012, Minsk, Belarus;

E-mail: selforg@newman.bas-net.by $\vec{x}_{i}^{(m)}=\left(\eta_{i}, \eta_{i+p}, \ldots, \eta_{i+(m-1) p}\right)$,

where $\eta(j \Delta t)=\eta_{j}, j=1,2, \ldots, N$ is a TS of a kinetic variable measured from the CS with a fixed time interval $\Delta t$, $\tau=p \Delta t$ is the delay time, $\mathrm{p}$ is an integer. The points $\vec{x}_{i}^{(m)} \subset R^{m}, R^{m}$ is an Euclidean phase space with a dimension $m, i=1,2, \ldots, L^{(p, m)}$, the common quantity of the attractor points is given by $L^{(p, m)}=N-p(m-1)$. In accordance with (1), phase trajectories forming the attractor $R_{T}^{m}$ can be represented as a superposition of $p$ rarefied sequences $\mathrm{X}_{1}, \mathrm{X}_{2}, \ldots, \mathrm{X}_{p}$ shifted by one sample with respect to each other, those are defined as $\mathrm{X}_{s}=\left\{\vec{x}_{s+p(k-1)}^{(m)}\right\}_{k=1}^{L_{s}^{(p, m)}}$.

As it was recently shown, rarefying on attractor points is reasonable at numerical simulation of fractal-topological analysis [9] for creating a subset of points with decorrelated components. Otherwise, using points that are too close together in time leads to aggravating accuracy of the fractal analysis. So, we use only one $X_{p}$ for numerical experiments and denote the components of $\Psi_{p}$ for brevity as $\Psi_{p}=\left\{\xi_{1}, \xi_{2}, \ldots, \xi_{N_{p}}\right\}$.

At the realization of Grassberger - Procaccia algorithm (GPA) $[1,5]$, the center of a cell (m-dimensional ball with a radius l) just coincides with a point $\vec{x}_{i}^{(m)}$ of a phase trajectory. At the same time, the direct use of the GPA leads to a multiple mutual covering of such cells that has the explicit difference in comparison with the initial consideration of a fractal analysis, namely regarding the boxcount method where segmentation cells do not cover each other at all [4]. So, additional rarefying at the fractal analysis 
in this section allows to reduce the above mentioned covering.

Since $r_{i j}=r_{j i}$ and $r_{i i}=0$ for distances on $\mathrm{X}_{p}$, so the square distance matrix $L \times L$ can be reduced to the upper triangle one $\widetilde{\mathfrak{R}}^{(m)}$ as follows:

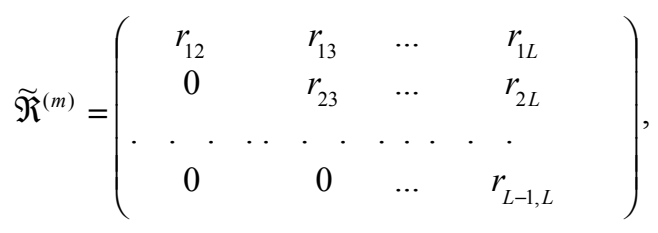

where we denote $L=L_{p}^{(p, m)}$, while distances are calculated as

$$
r_{i j}=\left[\sum_{k=1}^{m}\left(\xi_{i+k-1}-\xi_{j+k-1}\right)^{2}\right]^{\frac{1}{2}} .
$$

Taking into consideration cyclic (in other words, sift-byone coordinate property) within one sequence $\mathrm{X}_{s}$ (that can be obtained from (3), see ref. [10]), one can write the formula for transformation of distances along the diagonals of (2)

$q_{i+1, j+1}=q_{i, j}-\left(\xi_{i}-\xi_{j}\right)^{2}+\left(\xi_{m+i}-\xi_{m+j}\right)^{2}$,

where $q_{i, j}=\left(r_{i j}\right)^{2}$. The proposed algorithm for calculation of a fractal dimension implies detailed calculation of an Euclidean norm (3) for the upper row of (2) only. All other rows are calculated along the diagonals of (2) taking into account the following expression being obtained from (4):

$q_{i+1, i+\delta+1}=q_{i, i+\delta}-\left(\xi_{i}-\xi_{i+\delta}\right)^{2}+\left(\xi_{m+i}-\xi_{m+i+\delta}\right)^{2}$,

where $\delta=j-i$ is constant for all elements of the certain lateral diagonal: $\delta=1,2, \ldots, L-2$. In this process, one passes successively through all diagonals of (2) (both main and lateral ones) in the order of decreasing a total number of their elements. As usually $L>1000$ for supplying comprehensive statistical investigation, so proposed approach provides that calculation complexity for $r_{i j}$ determination practically does not depend on $m$ (because the main quantity of computational operations for calculation of distances is implemented by means of (5)), in contrast to direct application of the box-count method or the GPA $[1,5]$.

Such modification has the advantage comparing the introduction of the "supreme" norm [11]. (where the replacement of a vector norm by the largest coordinate occurs) because in our case the norm of a vector does not change and therefore we use the real distances on phase trajectories that provides the better accuracy of calculation process.

The further realization of proposed approach is as follows. After calculation of all distances composing the matrix $\widetilde{\mathfrak{R}}^{(m)}$, samples for $l$ are obtained as $l_{k+1}=l_{k}+\Delta l$, where $k=1,2, \ldots, N_{l}$ and initial size is chosen as

$l_{1}=a_{1}+n_{1} \Delta l$,

the interval value is $\quad \Delta l=\frac{a_{2}-a_{1}}{N_{1}} ; \quad a_{1}=\min _{i, j}\left\{r_{i j}\right\}$; $a_{2}=\max _{i, j}\left\{r_{i j}\right\}$. For calculation of correlation integral $C(l)^{3}$, one can use the property that $C(l)$ is just averaged probability of the event that the distance between two arbitrary chosen points of $R_{T}^{m}$ is less than $l$. So, the following is valid

$C(l)=\frac{n(l)}{L_{r}}$,

where $n(l)$ is the number of distances involved by (2) (except zeros) whose value is less than $l$, and the value $L_{r}=\frac{L(L-1)}{2}$ is the total number of nonzero elements in (2). According to the proposed computer algorithm, $n(l)$ is calculated successively along every lateral diagonal simultaneously with calculation of $r_{i, i+\delta}$ by (5). Estimation of $n_{\delta}\left(l_{k}\right)$ within a certain diagonal includes the following stages allowing minimization of comparison operations: (i) sorting the string $\left\{r_{i, i+\delta}\right\}$ in the order of increasing their elements resulting in $\left\{r_{i, i+\delta}^{(s o r t)}\right\}$; (ii) calculation of a number of distances $n_{\delta}\left(l_{k}\right)$ whose magnitude is less than $l_{k}$ that is implemented by the following recurrent relationship (see the code in the Appendix below, the part of the program called as THE COUNT OF HITS ON DIAGONALS):

$n_{\delta}\left(l_{k+1}\right)=n_{\delta}\left(l_{k}\right)+\Delta n_{\delta}\left(l_{k}\right)$,

where $n_{\delta}\left(l_{1}\right)$ is found by successive comparison of $r_{i, i+\delta}^{(s o r t)}$ and $l_{1}$, while at estimation of $n_{\delta}\left(l_{2}\right)$ the comparison process begins from $i=n_{\delta}\left(l_{1}\right)+1$ (similarly this process is running for subsequent values of $l_{k}$ ) that reduces a number of operations. When all $L-1$ diagonals have been passed, the total quantity of hits is $n\left(l_{k}\right)=\sum_{\delta} n_{\delta}\left(l_{k}\right)$.

The parameters $n_{1}, N_{l}, N_{1}$ in (6) are chosen so that the following property would be valid at the range of changing $l: \ln C(l) \propto \ln (l)$, and hence the correlation dimension is given by

$D_{2}=\frac{\Delta \ln C(l)}{\Delta \ln (l)}$.

It should be noted that the calculation complexity of developed algorithm increases with enlarging time series length $\mathrm{N}$ as $v^{2}$, where $v=\frac{N+\Delta N}{N}$ describes the growth of 
$N$ (because of quadratic growth of a number of elements in (2). This property is characteristic of all methods operating with $r_{i j}$ involved by the attractor. But due to minimization of operations according to (5), enlarging $\mathrm{N}$ results in far less calculation complexity than in a case of direct use of the correlation integral method, inasmuch as the initial calculation complexity of proposed algorithm is reduced due to rarefying TS and application of the localized approach to calculation of distances.

\section{NUMERICAL SIMULATIONS}

As a model TS, we use the TS obtained after discretization of the Mackey-Glass equation by the direct Euler approximation

$\xi_{i+1}=\beta \xi_{i}+\Delta t \phi\left(\xi_{i-\widetilde{n}}\right)$,

where $\quad \beta=1-\Delta \mathrm{t} b ; \quad \tilde{n}=\frac{\Delta T}{\Delta t} ; \quad \phi\left(\xi_{i-\widetilde{n}}\right)=\frac{a \xi_{i-\widetilde{n}}}{1+\left(\xi_{i-\widetilde{n}}\right)^{c}}, \quad$ and parameters in it and initial conditions have been fixed as well as in $[5,6]$. The equation (10) really provides the behavior whose complexity and chaos measures increase with the delay time $\Delta T$ (see ref. [6] where calculated TS are represented). In this paper, we also construct phase trajectories of $R_{T}^{3}$ by (1) at $\tau=6, \Delta t=0.5$ (Fig. 1). Evidently, for $\Delta T=17$ where the system is at the beginning of its chaotic behaviour (since transition to chaos proved to occur at $\Delta T=16.8$ ), the evolution of the system proceeds on the quasiperiodic trajectories. From the other hand, at $\Delta T=100 \quad$ (Fig. 1b) the trajectories acquire irregular behaviour and after elapsing a certain time fill some restricted region of the phase space. Thus, in spite of its approximate character, this construction really reflects transition to the fully developed chaos with increasing dimension.

Next, we implemented the calculation of the fractal dimension by (1)-(10) for $\Delta T=30$ and $\Delta T=100$ using (for better accuracy) the following parameters of (10) discretization: $\Delta t=0.25, p=24$, while $N_{p}=5000$ and

20000 for integer dimensions $m_{0}=6$ and 18 respectively.

The $N_{p}$ value defines the length of TS really used for calculations (i.e. after rarefying), while initial length $N=p N_{p}$. The results of calculations are shown in Figs.

$(2,3)$. One can see that $\log -\log$ renormalization really linearizes the dependence $C(l)$ versus $l$. The values of $D_{2}$ determined from calculated dependencies are as follows: $D_{2}=2.9 ; 9.4$ respectively for $\Delta T=30$ and 100 , that is similarly to results obtained in ref [5].

The values of parameters in (6) for our computer experiments were $N_{1}=1000, N_{l}=50, n_{1}=5$. Evidently the boundaries of fractal dimensions tend to enlarge with growth of $\Delta T$ as follows from Fig. (3).
At the computer realization of the code (see the Appendix), the largest time was expended for calculation of the initial TS (the part CALCULATION OF THE MODEL TIME SERIES), approximately $90 \%$. At growing $N$, we detected that the time of fractal analysis grows more slowly than the time $t_{T S}$ of TS calculation. Since $t_{T S} \propto N$, one can conclude that the time of fractal analysis using this code grows with $N$ even more slowly than linearly.
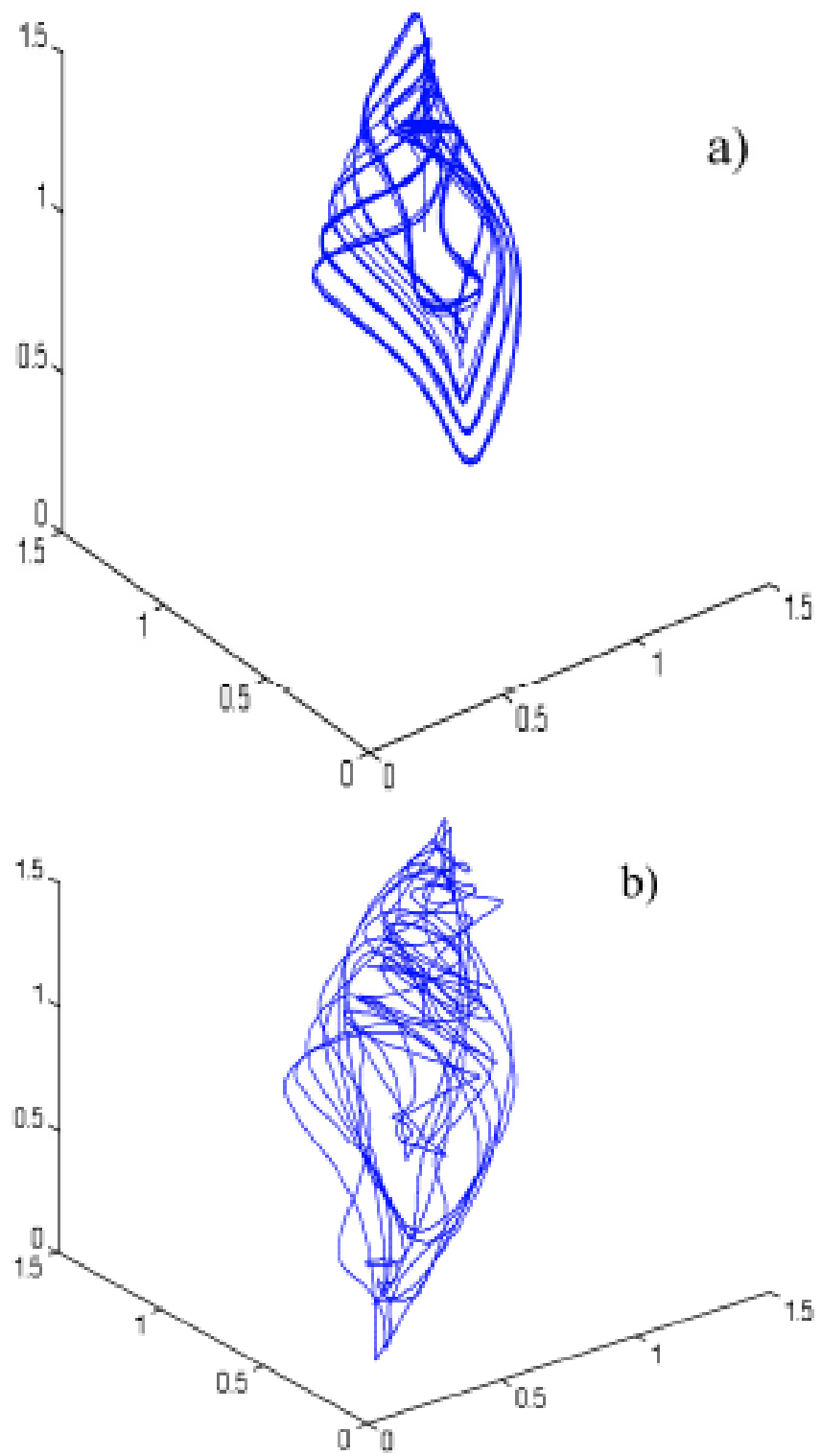

Fig. (1). Phase trajectories of the chaotic attractor for the system with nonlinear time delay: (a) $\Delta T=17$; (b) $\Delta T=100$.

The time of additional calculations (i.e., the fractal dimension itself through distances comparison as described in section 2) is reduced due to localized properties along diagonals in (2). Such localized approach is used in the code twice: at calculation of EPS and probabilities of points vicinity. It is worth noting that permutation properties of cycles in the part "THE COUNT OF HITS ON DIAGONALS" are also used that leads to additional reduction of computer resources. 


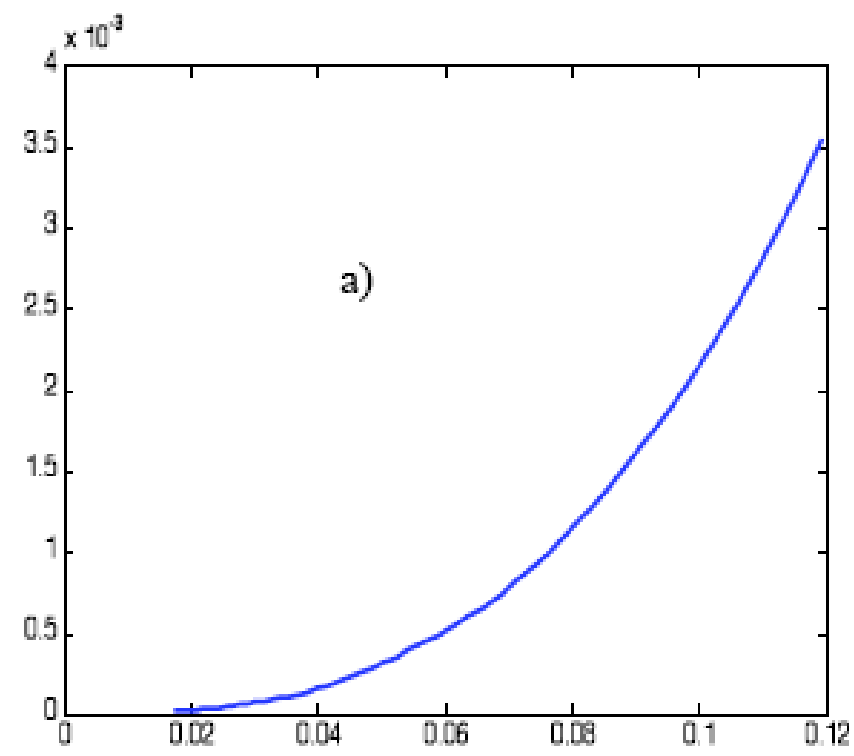

$Q_{M}=\prod_{j=0}^{M-1}\left(D_{M-j-1} V\right)$,

while its components are $\quad V=U F$; $D_{j}=\operatorname{diag}\left\{\psi_{\tilde{j} \tilde{n}}, \psi_{j \tilde{n}+1}, \ldots, \psi_{(j+1) \tilde{n}-1}, \beta^{\tilde{n}+1}\right\}, \quad \psi_{k}=\Delta t \frac{d \phi\left(\xi_{k}\right)}{d \xi_{k}}$, $U$ and $F$ are the binary matrices: lower triangle matrix $(U)_{i, j}=\left[\begin{array}{l}1, \text { if } i \geq j ; \\ 0, \text { otherwise; }\end{array}\right.$

and permutation matrix providing the cyclic shift [12].
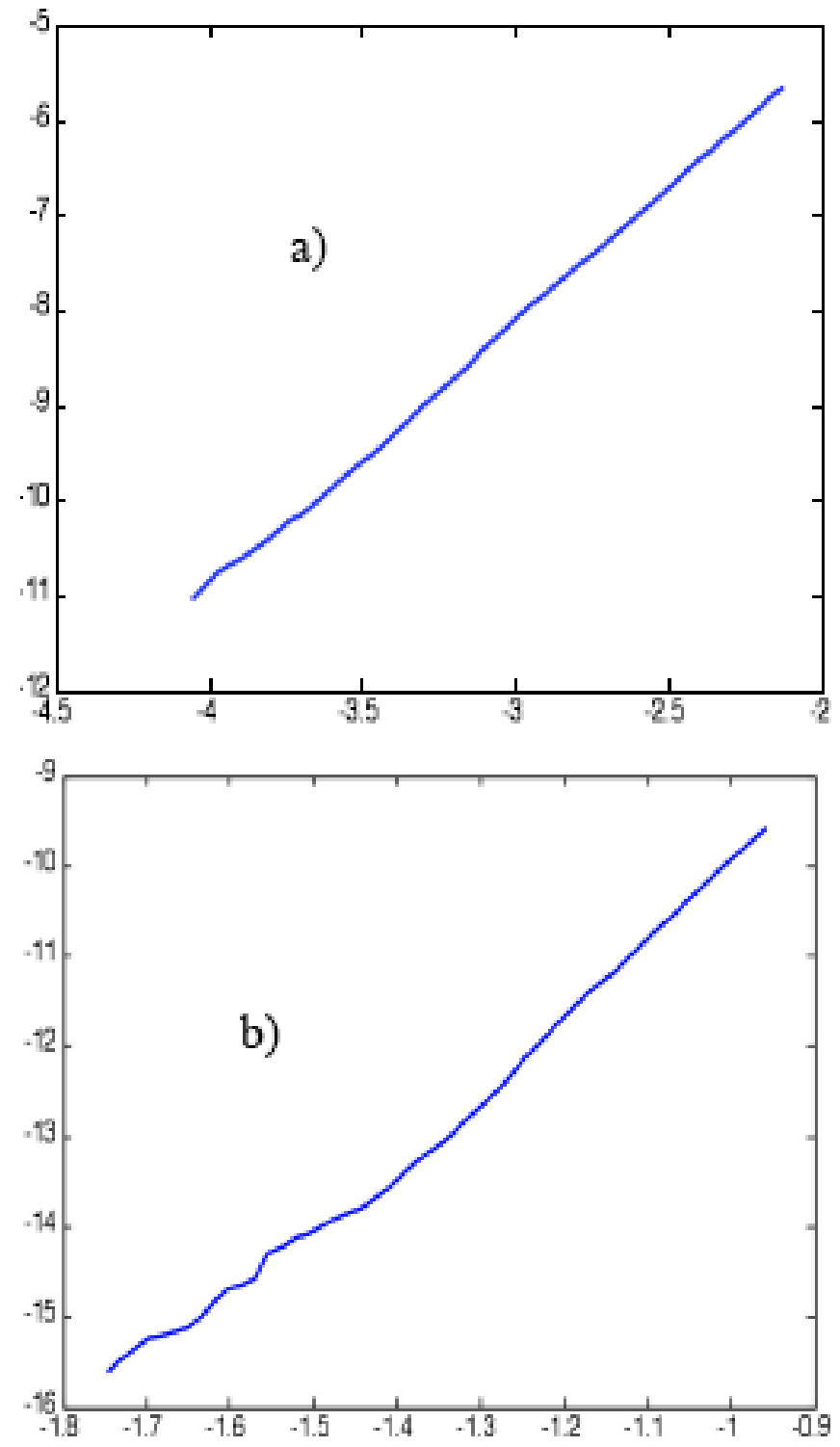

Fig. (3). The plot of $\ln \mathrm{C}(l)$ vs $\ln l$ for different delay times (log$\log$ renormalization of dependencies displayed in Fig. (2)): a) $\Delta T=30 ;$ b) $\Delta T=100$.

At the numerical simulations of developed analytical scheme, $M=100$ and $\Delta \mathrm{t}=0.25$, while the corresponding derivatives have been calculated from the samples obtained of the TS using the relationship derived in ref. [6], $N=M \tilde{n}$. The complete spectrum of the characteristic exponents is 
shown in Fig. (4). These numerical results really prove that the quantity of positive characteristic exponents increases with $\Delta T$. Since $v_{k}$ are considered as topological invariants of the attractor [1], those can reflect the temporal evolution of the system and its averaged instability.
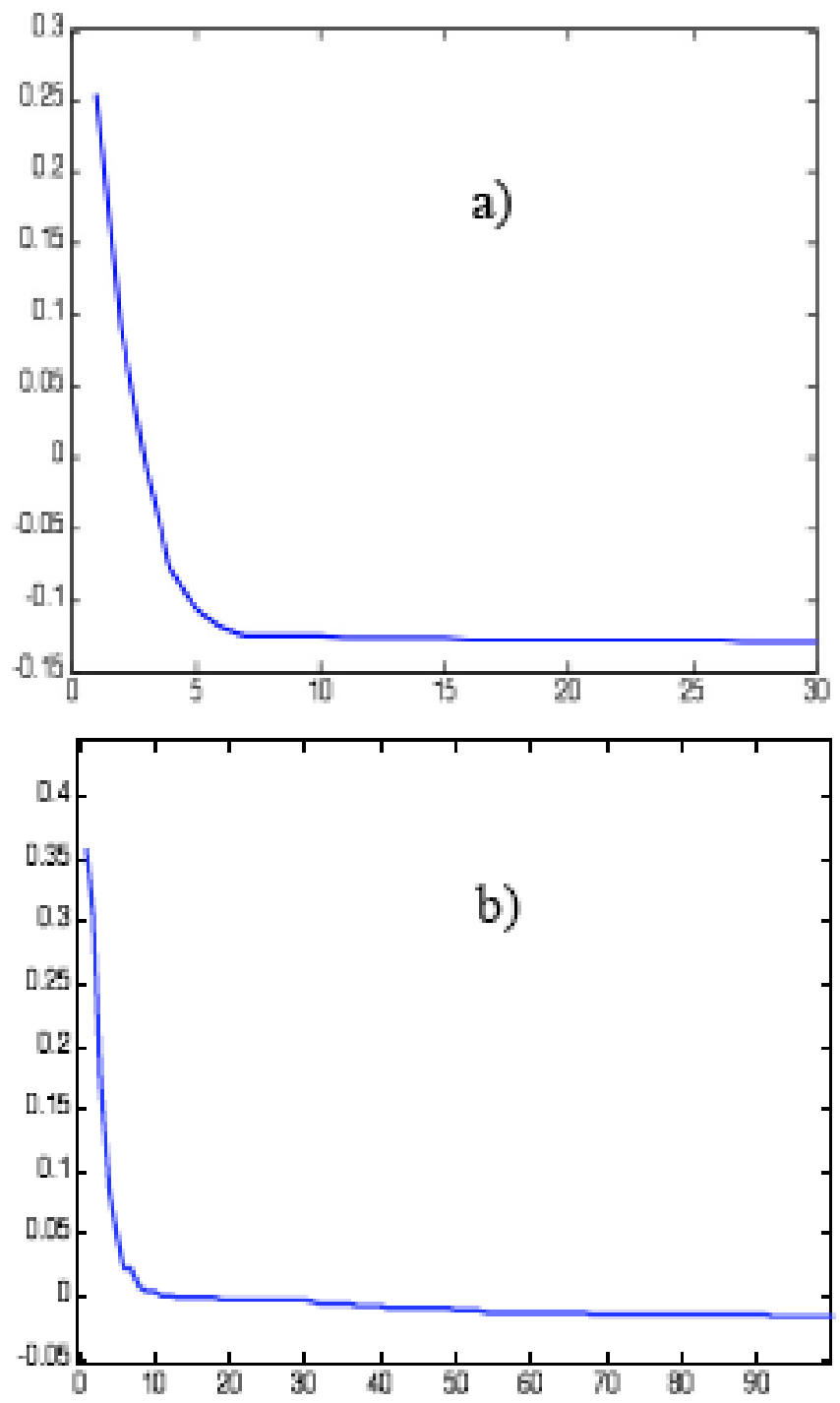

Fig. (4). The plot of the characteristic exponents $v_{k}:$ a) $\Delta T=30$; b) $\Delta T=100$; the displayed region of $k$ is restricted proportionally $\Delta T$.

Considering the functional matrix of the map $\vec{x}_{i}^{(m)} \rightarrow \vec{x}_{i+p}^{(m)}$, i.e. within a rarefied TS

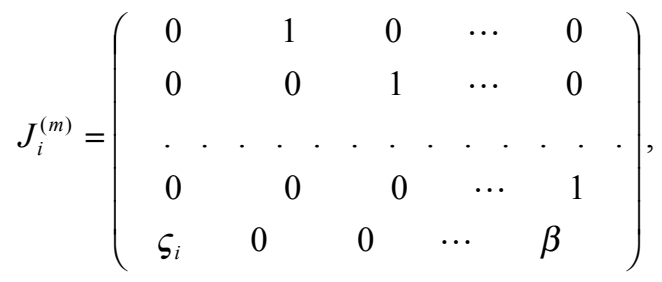

one can see that it reflects a shift by one sample and therefore is of Frobenius form, where $\zeta_{i}=\beta^{m p-(\tilde{n}+1)} \psi_{i}$. After numerical calculations of the eigenvalues of the inverse multiplication

$\bar{P}_{M}=\prod_{j=0}^{M-1} J_{M-j-1}^{(m)}$,

and its averaging, we detected that the accuracy of this approach of describing instability is less than used above (see (11) - (12)), because it provides less smooth curve of the spectrum and reduced quantity of characteristic exponents, for the reason of reduction of the map dimension.

One more approach that is worthy of exploration is the map corresponding to the minimal shift along the phase trajectory (1), namely $\vec{x}_{i}^{(m)} \rightarrow \vec{x}_{i+1}^{(m)}$. Taking into account (1), (10), one can show similarly to ${ }^{6}$ that the functional matrix of the map is of the triangle Toeplitz form, i.e.

$T_{i}^{(m)}=$

$\left(\begin{array}{cccccc}\beta & 0 & \cdots & 0 & 0 \\ \beta^{(p+1)} & \beta & \cdots & 0 & 0 \\ \cdot \cdot \cdot \cdot \cdot \cdot \cdot \cdot \cdot & \cdot & \cdot & \cdot & \cdot & \cdot \\ \beta^{(m-1) p+1} & \beta^{(m-2) p+1} & & \beta & 0 & \\ \bar{\zeta}_{i} & \beta^{(m-1) p+1} & \cdots & \beta^{(p+1)} & & \beta\end{array}\right)$,

where $\bar{\zeta}_{i}=\beta^{l(\beta)} \psi_{i}$, while $\beta^{l_{(\beta)}} \psi_{i}$ does not depend on the point number. The inverse multiplication

$\bar{T}_{M}=\prod_{j=0}^{M-1} T_{M-j-1}^{(m)}$,

can be calculated for such a case in the exact analytical form, namely

$\bar{T}_{M}=\beta^{M} B^{(p)} S^{(M)}\left(B^{(p)}\right)^{-1}$

where

$S^{(M)}=\prod_{j=0}^{M-1} \Delta_{M-j-1}^{(m)} \quad$ is the multiplication of the triangle matrixes

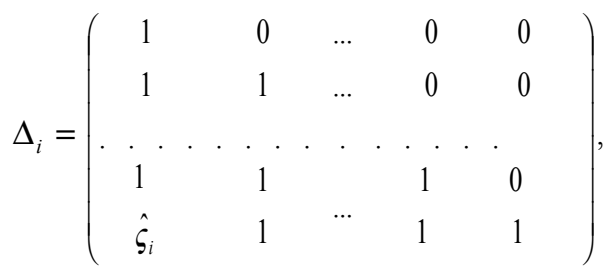

and $\hat{\zeta}_{i}=\bar{\zeta}_{i} / \beta^{(m-1) p+1}$, while the matrix $B^{(p)}$ is the diagonal one consisting of the polynom on $\beta$. But using this approach, the characteristic exponents corresponding to integrated map can not be expressed through eigenvalues, and singular values calculation with additional transformations would be useful. For solving this problem through eigenvalues, the shift properties as in (13) should be taken into account. 


\section{CONCLUSIONS}

Thus, the algorithms based on temporal localization regarding points of attractor is developed. It allows the essential reduction of required experimental resources at the expense of minimization of computational operations. Numerical simulations with the CS containing the nonlinear delayed feedback described by a nonlinear differential equation with retarded argument (that is very typical for cybernetics and automatic control) proved high accuracy of determination of topological characteristics (the fractal dimension and characteristic exponents). The represented algorithm is applicable for the sake of characterization of complex systems under investigation. In particular, within tasks of modeling of turbulent flows the method can be applied for the characterization of the fully developed turbulence, mainly for estimation of turbulent energy dissipation and the complexity of the process.

It should be noted that the developed algorithms can be easily generalized for the calculation of all spectrum of fractal dimensions (e.g., in generalized correlation integral method [13]). Then the approach described in section 2 can be applied for calculation of distances along the diagonals, those should be retained in storage for further calculation (at successive enlargement of the order of related moments defining the averaged probabilities).

\section{APPENDIX: THE CODE FOR FRACTAL ANALYSIS}

The program is written and used for the MATLAB V software (see below). The program implies the processing of diagonals of (2) in the order of enlarging a number of elements involved by each diagonal. This allows the successive enlargement of the working space for every diagonal array and proper sorting of the array. At this condition, avoiding excessive elements is attained because of complete replacement of diagonal array at transition to every subsequent diagonal.

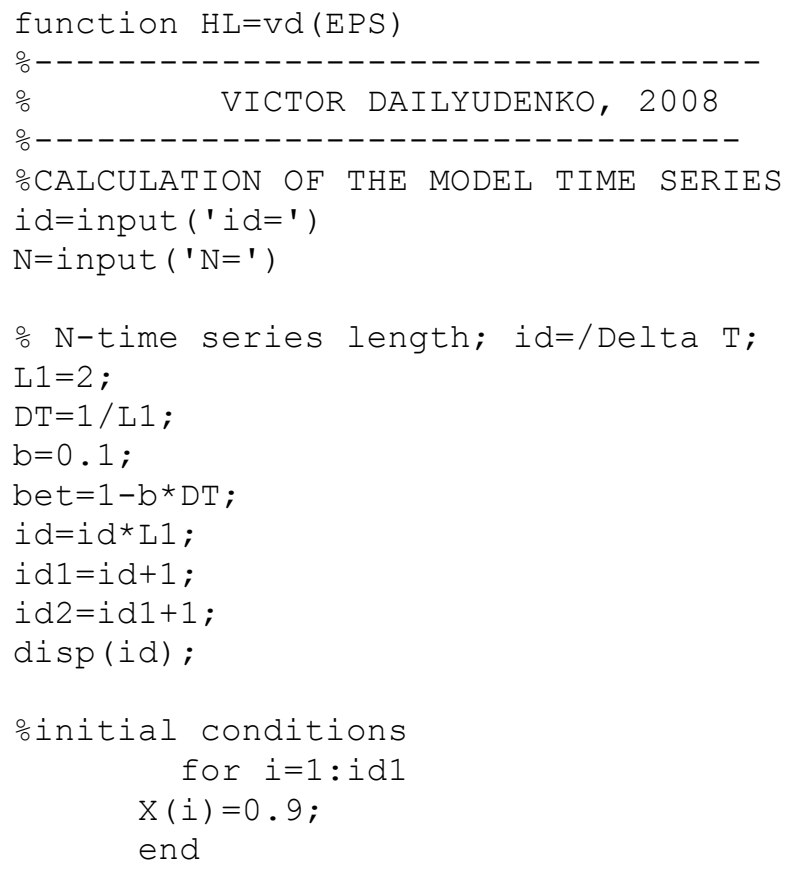

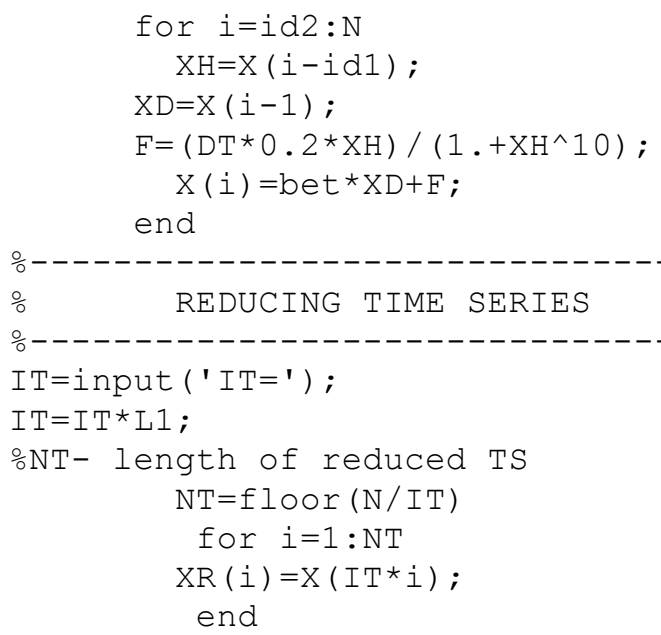




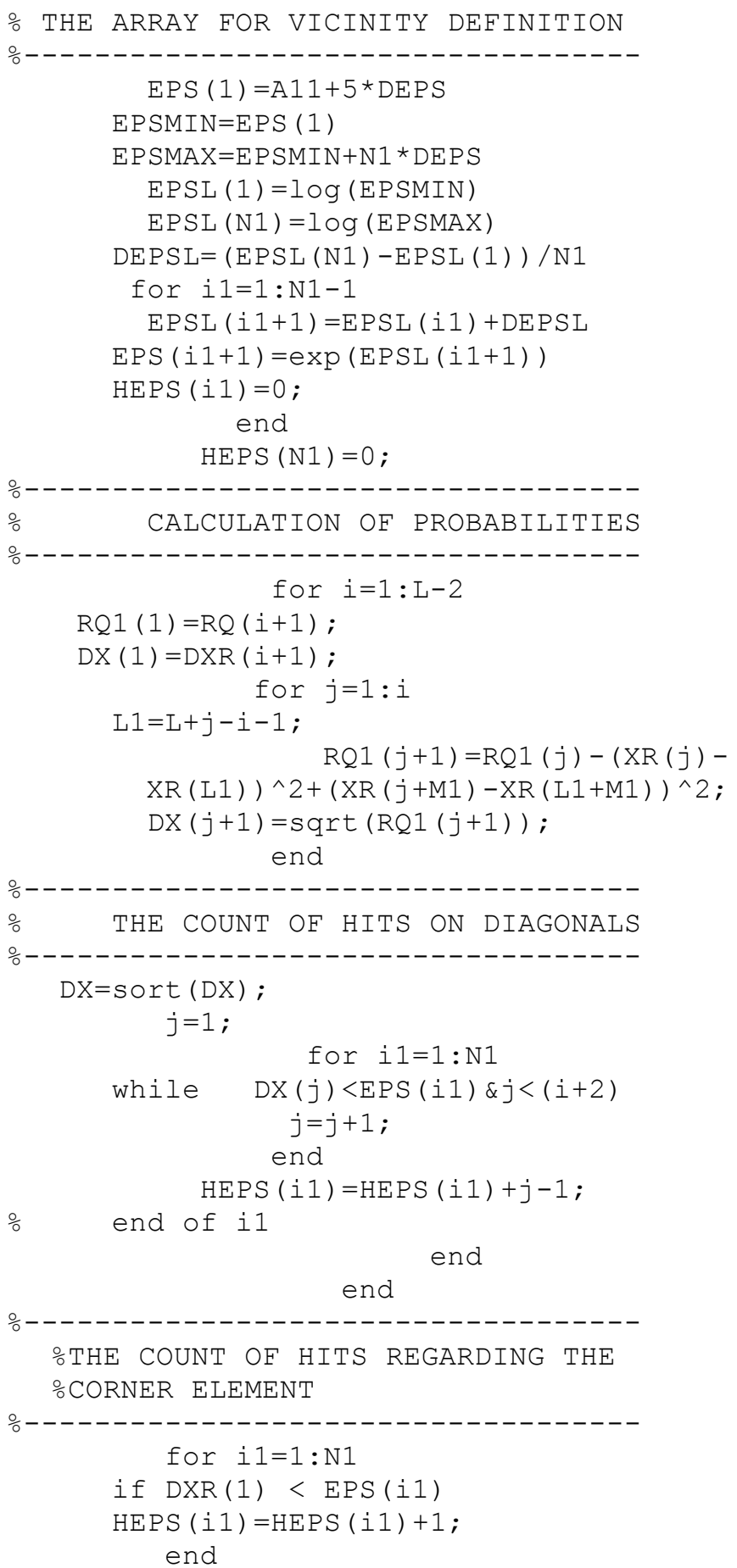

H1 (i1)-probability of a hit into oEPS(i1)-vicinity of an attractor opoint

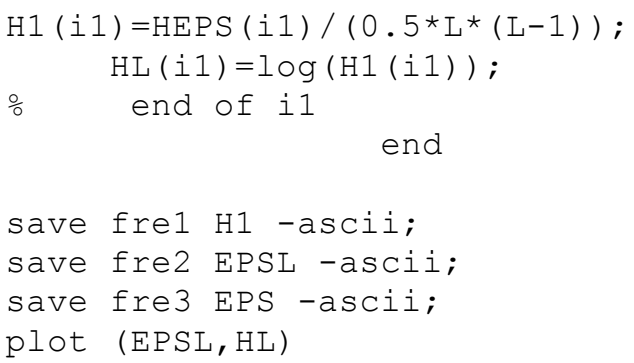

\section{REFERENCES}

[1] A.G. Crilly, R.A. Earnshaw, H. Jones, Eds., Applications of Fractals and Chaos. Springer-Verlag: Berlin, 1993.

[2] L. Telesca, V. Lapenna, M. Macchiato, "Mono- and multifractal investigation of scaling properties in temporal patterns of seismic sequences", Chaos Solitons Fractals, vol. 19, pp. 1-15, Feb. 2004.

[3] J. Wang, X. Ning, Y. Chen, "Multifractal analysis of electronic cardiogram taken from healthy and unhealthy adult subjects", Phys. $A$, vol. 323, pp. 561-568, May 2003.

[4] L.M. Pecora, T.L. Carroll, D.J. Marr, "Fundamentals in synchronization in chaotic systems, concepts, and applications", Chaos, vol. 7, pp. 520-543, June 1997.

[5] J.D. Farmer, J.J. Sidorowich, "Predicting chaotic time series", Phys. Rev. Lett., vol. 59, pp. 845-849, May 1988.

[6] V.F. Dailyudenko, "Lyapunov exponents for complex systems with delayed feedback", Chaos Solitons Fractals, vol. 17, pp. 473-484, Apr. 2003.

[7] J. Guckenheimer, P. Holmes, Nonlinear Oscillations, Dynamical Systems, and Bifurcations of Vector Fields. Springer-Verlag: Berlin, 1983.

[8] F. Takens, "Detecting strange attarctors in turbulence", in Dynamical Systems and Turbulence, Lecture Notes in Math. Springer: Berlin, vol. 898, 1981, pp. 366-381.

[9] A.M. Albano, A. Passamante, M.E. Farrell, "Using higher-order correlations to define an embedding window", Phys. D, vol. 54, pp. 85-97, June 1991.

[10] V.F. Dailyudenko, "Nonlinear time series processing by means of ideal topological stabilization analysis and scaling properties investigation", in SPIE's Conference on Applications and Science of Computational Intelligence II, 1999, pp. 108-119.

[11] A.V. Holden, J. Hyde, H. Zhang, "Computing with Unpredictable: Chaotic Dynamics and Fractal Structure of the Brain", in Application of Fractals and Chaos. R.A. Earnshaw, Ed., SpringerVerlag: Berlin, 1993, pp. 215-235.

[12] V.F. Dailyudenko, "The integrated and local estimations of instability for a class of autonomous delay systems", Chaos, Solitons Fractals, vol. 30, pp. 759-768, Aug. 2006

[13] L.V. Meisel, M.A. Johnson, "Multifractal analysis of imprecise data: Badii-Politi and correlation integral approach", Phys. Rev. E, vol. 50, pp. 4214-4219, Apr. 1994.

This is an open access article licensed under the terms of the Creative Commons Attribution Non-Commercial License (http://creativecommons.org/licenses/by$\mathrm{nc} / 3.0 /$ ) which permits unrestricted, non-commercial use, distribution and reproduction in any medium, provided the work is properly cited. 